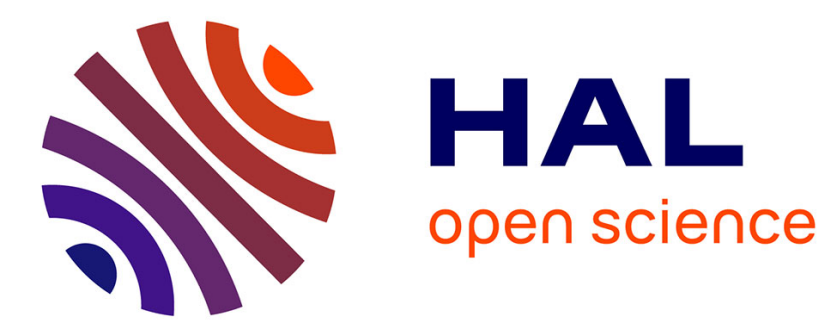

\title{
Coexistence of ICN and IP networks: an NFV as a service approach
}

Boubakr Nour, Fan Li, Hakima Khelifi, Hassine Moungla, Adlen Ksentini

\section{To cite this version:}

Boubakr Nour, Fan Li, Hakima Khelifi, Hassine Moungla, Adlen Ksentini. Coexistence of ICN and IP networks: an NFV as a service approach. GLOBECOM 2019: IEEE Global Communications Conference, Dec 2019, Waikoloa, HI, United States. pp.1-6, 10.1109/GLOBECOM38437.2019.9013881. hal-02266947

\section{HAL Id: hal-02266947 https://hal.science/hal-02266947}

Submitted on 17 Aug 2019

HAL is a multi-disciplinary open access archive for the deposit and dissemination of scientific research documents, whether they are published or not. The documents may come from teaching and research institutions in France or abroad, or from public or private research centers.
L'archive ouverte pluridisciplinaire HAL, est destinée au dépôt et à la diffusion de documents scientifiques de niveau recherche, publiés ou non, émanant des établissements d'enseignement et de recherche français ou étrangers, des laboratoires publics ou privés. 


\title{
Coexistence of ICN and IP Networks: An NFV as a Service Approach
}

\author{
Boubakr Nour*, Fan $\mathrm{Li}^{*}$, Hakima Khelifi ${ }^{\ddagger}$ Hassine Moungla ${ }^{\S \llbracket}$, and Adlen Ksentinill \\ ${ }^{*}$ School of Computer Science, Beijing Institute of Technology, Beijing, China \\ $\ddagger$ School of Information and Electronics, Beijing Institute of Technology, Beijing, China \\ ${ }^{\S}$ Université de Paris, LIPADE, F-75006 Paris, France \\ IIUMR 5157 CNRS, Telecom SudParis, Institut Polytechnique de Paris, Palaiseau 91120, France

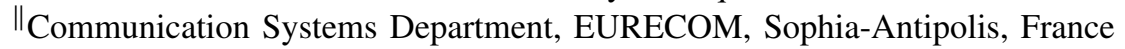 \\ Email: \{n.boubakr, fli, hakima\}@bit.edu.cn, hassine.moungla@parisdescartes.fr, adlen.ksentini@eurecom.fr
}

\begin{abstract}
In contrast to the current host-centric architecture, Information-Centric Networking (ICN) adopts content naming instead of host address and in-network caching to enhance the content delivery, improve the data distribution, and satisfy users' requirements. As ICN is being incrementally deployed in different real-world scenarios, it will exist with IP-based services in a hybrid network setting. Full deployment of ICN and total replacement of IP protocol is not feasible at the current stage since IP is dominating the Internet. On the other hand, redesigning TCP/IP applications from ICN perspective is a timeconsuming task and requires a careful investigation from both business and technical point of view. Thus, the coexistence of ICN and IP is one of the suitable solutions. Towards this end, we propose a simple yet efficient coexistence solution based on Network Function Virtualization (NFV) technology. We define a set of communication regions and control virtual functions. A gateway node is used as an intermediate entity to fetch and deliver content over regions. The simulation results show that the proposed approach is valid and allow content fetching and delivering from different ICN and/to IP regions in an efficient manner.
\end{abstract}

\section{INTRODUCTION}

Network Function Virtualization (NFV) [1] is one of the essential technologies to realize next-generation networks (e.g., 5G networks). It aims to build complex virtual network functions on top of a virtualized infrastructure, manage and orchestrate their processing in order to afford seamless connectivity and communication. On the other hand, the huge coming of the Internet of Things (IoT) generates a massive growth of content due to the large number of connected devices. the current Internet ecosystem is facing immense changes in the applications' design and users' requirements. Indeed, the system is shifting from a connectivity-based to content-oriented communication. Toward this, Information-Centric Networking (ICN) [2] has been proposed as an alternative communication model to replace the current IP protocol.

ICN uses the content name as the main network element to drive communication [3]. It aims at decoupling the content from its original location and implementing content-based security [4]. Hence, the network layer becomes aware of content delivery by applying in-network caching. To realize the vision of ICN, various architectures have been implemented such as Content-Centric Networking $(\mathrm{CCN})$ and Named Data Net- working (NDN) [5]. These architectures are receiver-driven, use hierarchical names to identify content, and follow interestdata exchange model. The simple and clean design of ICN promotes it as a suitable communication model on different domain applications such as the Internet of Things [6]-[8], vehicular networks [9]-[11], 5G networks [12], etc.

ICN enhances the content distribution and availability in the network and overcomes single-point failure issue [13]. Consequently, it promises to implement efficient communication compared to IP protocol. However, the latter is dominate communication protocol on the Internet where various economic and technical factors should be considered before migrating from IP to ICN. For the economic factors, service providers and companies need to study the cost of migration and provide in-network storage [14]. While technical factors concentrate on the way how ICN can be deployed, i.e. either by (a) complete replacement of IP protocol, (b) running ICN on top of a transport protocol such as Transmission Control Protocol (TCP) or User Datagram Protocol (UDP), or (c) coexistence with IP protocol.

The main motivation behind this work is to address how to adopt ICN while being able to interoperate with IP networks. In fact, the optimal approach for existing networks is to focus not only on transition methods but also on coexistence. In a coexistence environment, some nodes can communicate using IP protocol, while others using ICN model. Moreover, both IP- and ICN-based services can exist and exchange content without the need to re-design the whole applications. Hence, in this paper, we study the existence of IP and ICN networks and propose a simple yet efficient NFV as a Service (NFVaaS) approach to retrieval/deliver content from/to ICN or IP nodes. We divide the network into IP and ICN regions based on the used protocol and define a set of virtual control functions to ensure a seamless content retrieval between regions. A gateway node who is able to communicate using both protocols is used as an intermediate node during the communication. The proposed solution does not require any packet conversation rules or extra overhead, which is proven via simulation.

The rest of the paper is organized as follows. Section II reviews ICN deployment modes, existing solutions, and challenges. Section III details the proposed approach including the 


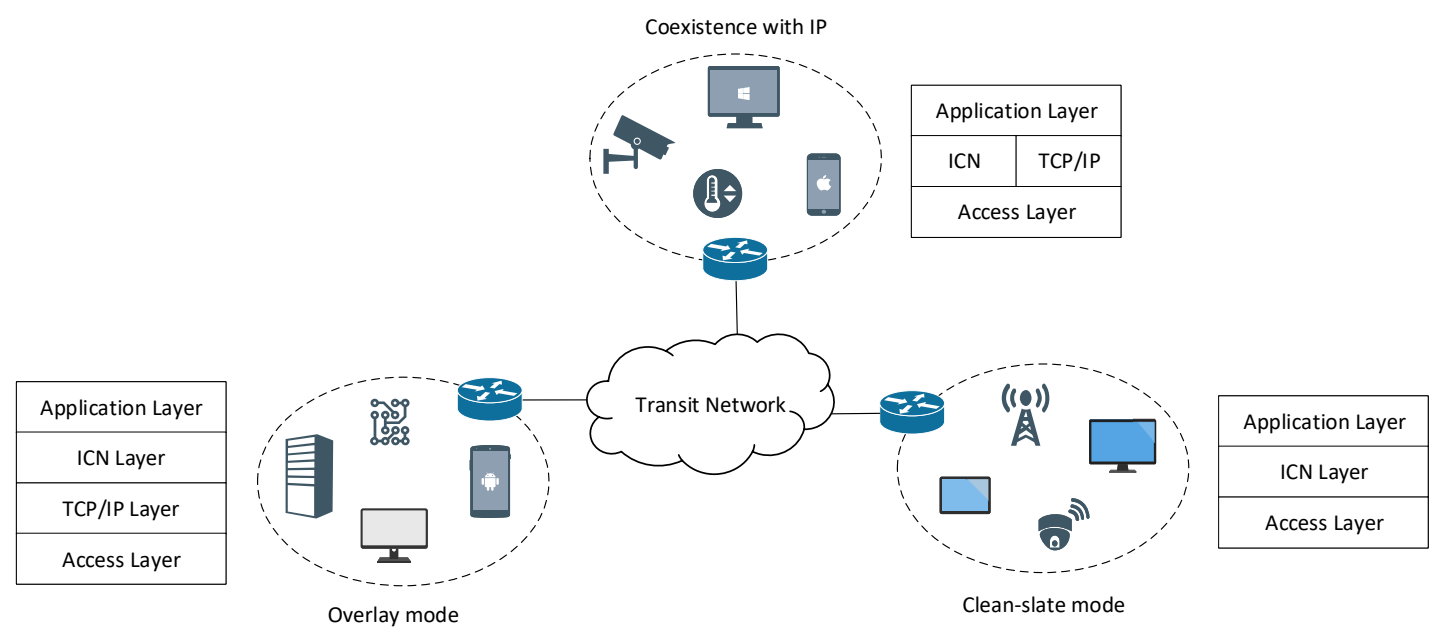

Fig. 1: Overview of ICN deployment.

architecture elements, control functions, and content delivery schemes. Section IV provides simulation results. Section V discusses different issues and challenges that face IP-to-ICN migration. Finally, Section VI concludes the paper.

\section{ICN Deployment: Existing Solutions \& Challenges}

The practical deployment of ICN is one of the most important questions that should be addressed before ICN being widely used as a communication model in the Internet. We figure out three modes to deploy ICN, as illustrated in Figure 1, and detailed as follows.

\section{A. Clean-slate Mode:}

A clean-slate mode of ICN refers to a full replacement of IP protocol and a direct deployment of ICN over the access layer. All nodes in such an environment must communicate using ICN. This deployment mode is easier and less complex, where there are no requirements for communication with IPbased nodes. However, maintaining backward compatibility, global access, and connectivity is required with the current Internet.

\section{B. Underlay \& Overlay Mode:}

An ICN architecture can be deployed as an overlay protocol over an existing IP infrastructure, for example over TCP or UDP protocols, or an underlay protocol by running IP-based services on top of ICN. Both modes require an extra layer to manage and encapsulate ICN inside IP packets (or vice-versa). This may create more overhead and complexity in the network. H2020 POINT project $^{1}$ (IP-over-ICN networking) [15], [16] is an active European project that aims at running IP protocol over ICN.

For instance, Shailendra et al. [17] design an Overlay Information Centric Networks architecture that separates the data plane from the control plane. The control plane contains

\footnotetext{
${ }^{1} \mathrm{H} 2020$ : https://www.point-h2020.eu/
}

both naming and routing, while the data plane contains innetwork caching. The authors define ICN Manager Module, which is an extended version of the Domain Name System, for name resolution purposes. Trossen et al. [18] discuss the feasibility to run ICN over IP-based services. To achieve this goal, the authors design a publisher-subscriber communication architecture to place IP-based services on top of ICN-driven networks, and then, it can support different protocols such as HyperText Transfer Protocol (HTTP), Constrained Application Protocol (CoAP), and TCP as well as various data structures of the underlying protocols within the ICN network. Shannigrahi et al. [19] design a tunneling mechanism to allow a transparent usage of IP applications in ICN networks. The overall idea consists of attaching the IP address as a component in the naming scheme, provides both encapsulation and re-sequencing during the communication at the network gateway level.

\section{Coexistence with IP:}

As IP is the dominant protocol in the current Internet, ICN coexisting with IP is indispensable. Translation of IP address and ICN names is required to build such a coexistence.

Vahlenkamp et al. [20] propose to use Software-Defined Networking (SDN) to enable ICN on an existing IP network. An SDN controller installs the appropriate forwarding state for each ICN demands. Thus, nodes in the network can support both IP and ICN forwarding without the need to install the ICN stack. Similarly, Zuraniewski et al. [21] discuss how SDN can be used to adopt ICN. The authors combine OpenFlow and Extended Berkeley Packet Filters to enable an intelligent forwarding plane without consulting the central SDN controller for every datagram.

Moiseenko et al. [22] propose a coexistence mechanism that allows applications to use TCP transport protocol to carry packets over an ICN network rather than IP over ICN. This can be achieved by using a TCP/ICN Proxy entity to carry traffic between two TCP nodes via an ICN network. Refaei et al. [23] design an IP-to-NDN Gateway system to allow 


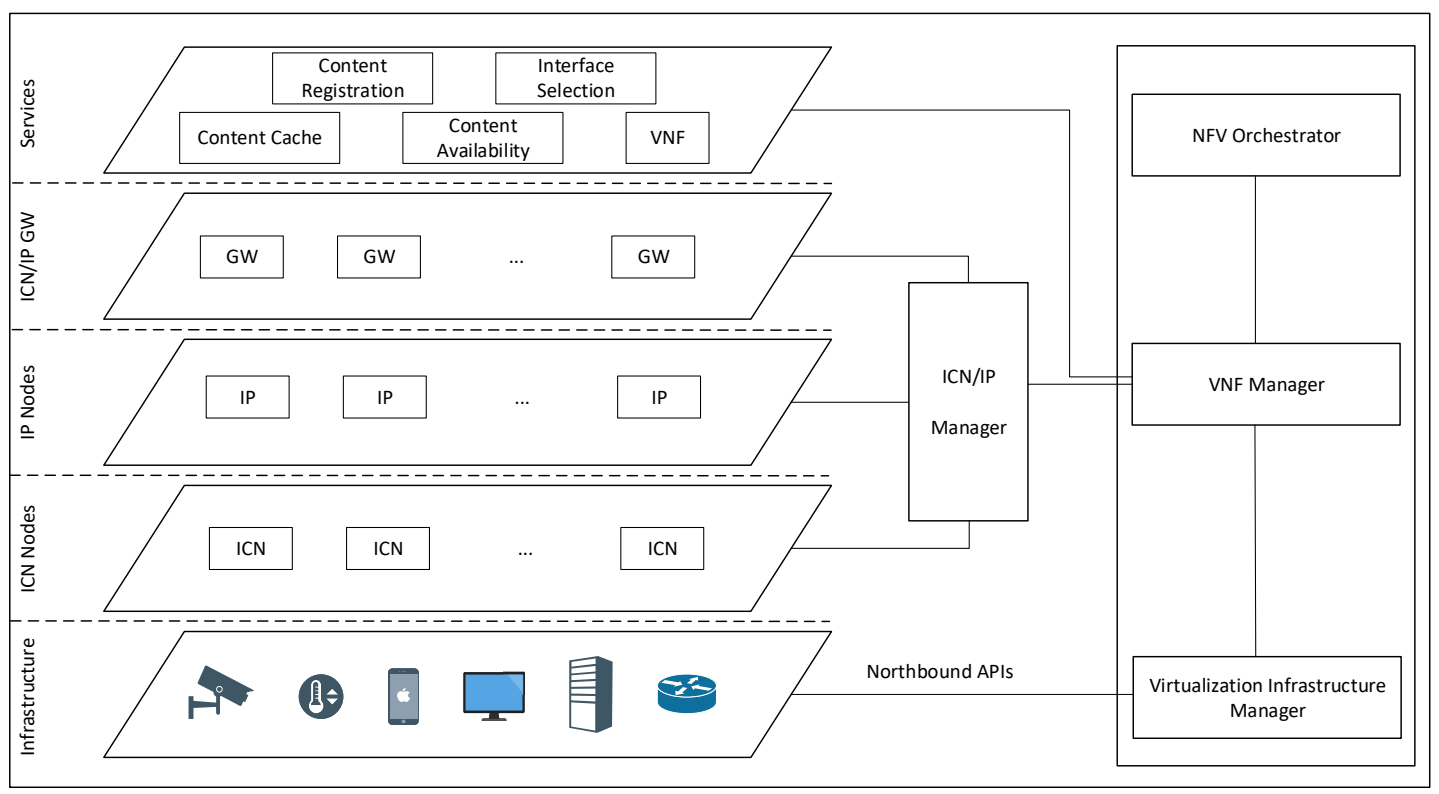

Fig. 2: Architecture Design.

IP-based applications to retrieve content from NDN networks without re-designing the transited packets. The system is based on a configuration file at the gateway level. This file tends to translate the packet header (from IP to ICN and vice-versa). However, the configuration file must include all possible headers and how to translate them, which is not a practical solution in large networks with dynamic traffic. Luo et al. [24] propose different migration schemes to translate TCP/IP packets into NDN format. The authors propose transaction mechanisms at three network layers: Internet, TCP, and Application layer. The overall idea consists of including all application and network-related information in the naming scheme (e.g., IP address), while the IP header is encoded into a specialized Interest packet (namely NotifyInterest). The main limitation of this solution is the scheme produces large names that affect network performance and scalability.

\section{NFV AS A SERVICE APPROACH}

The main objective of the proposed architecture, illustrated in Figure 2, is to integrate ICN-based communication to deliver and retrieve content to and from IP-based networks (and vice-versa). Indeed, it incorporates NFV as a Service to enable the coexistence of ICN and IP in a flexible and scalable manner.

\section{A. Architecture Elements}

The architecture contains both ICN- and IP-enabled devices. Both devices may create, request, and deliver content. The requested content can be delivered from the same region that uses the same protocol (i.e. IP-TO-IP, ICN-TO-ICN) or from other regions managed by other protocol (i.e. IP-TO-ICN, ICN-TO-IP). Thus, we define four types of region:

1) ICN REGION: contains nodes that use only ICN protocol. The communication is driven only by content name where the requested content is located in an ICN node (either original producer or a replica node).

2) IP REGION: contains nodes that use only IP protocol. The communication is directed using the destination host IP address.

3) ICN-TO-IP REGION: contains both ICN and IP nodes. The communication is initialized by an ICN node that requests content. The content is located in an IP REGION. Name-based routing rules are used in ICN REGION while IP addresses are used in IP REGION.

4) IP-TO-ICN REGION: is similar to ICN-TO-IP REGION with a difference that the communication is initialized by an IP node to retrieve content located in an ICN REGION.

Besides, a node that runs both ICN and IP protocols is considered as ICN/IP Gateway (ICN/IP GW). All of these nodes communicate with the ICN/IP Manager to monitor and report the resources usage at the node level. Moreover, the service layer contains a set of control functions that aim to enable communication and content traversing between different regions. These functions are managed by the VNF Manager entity - that also ensures the communication between different VNF instances, and handled by the NFV Orchestrator.

Finally, the architecture exposes a Northbound API to handle customers/users requests, and a Southbound API to enable communication between NFV Orchestrator and Virtualized Infrastructure Managers in order to satisfy the virtual resource requirements.

\section{B. Control Functions}

In order to provide a seamless and transparent coexistence, we define a set of virtual control functions.

- Content Registration Function: for each generated content, the system will register the content associated with 


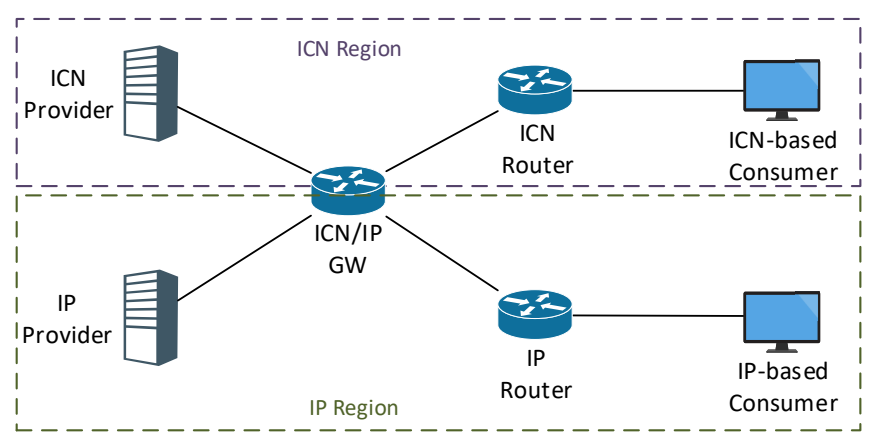

(a)

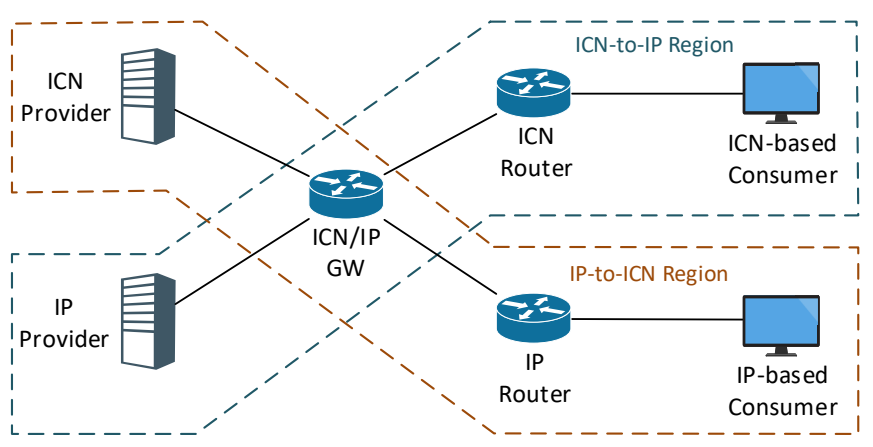

(b)

Fig. 3: Content Retrieval and Delivery Modes: (a) ICN/IP Region, (b) ICN-to-IP/IP-to-ICN Region.

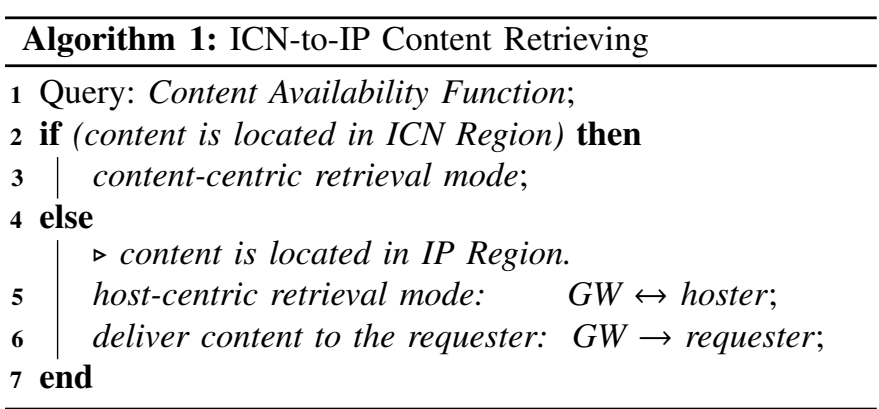

a unique name. If an ICN node was the producer then the registration will be bound with its name. Similarly, if the producer is an IP node, the registration will be done using the producer IP address and an on-fly generated flat name.

- Content Cache Function: since the IP layer cannot natively support in-network caching, we propose this function in order to enhance the content availability in IP REGION. An IP node may cache content temporarily. In such a scenario, it informs the VNF Manager about the cached content name associated with the host IP address.

- Content Availability Function: this function tries to provide information about the content availability (in which region and how to access it) by performing exact prefix match lookup on the list of registered content. It returns the content name if it is located in ICN REGION or IP address if it is located in IP REGION. By doing so, both the content discovery and delivery process will be enhanced.

\section{Content Retrieval \& Delivery Schemes}

In this work, we only provide essential functions to ensure ICN and IP coexistence. More functions can be defined by the network administrator to provide control, monitoring, and management functionalities.

Figure 3 depicts different content retrieval cases in a simple topology. In particular, we distinguish two base modes, as shown in Figure 3a: (a) content-centric retrieval mode: used in ICN REGIONS where only the content name is used to retrieval

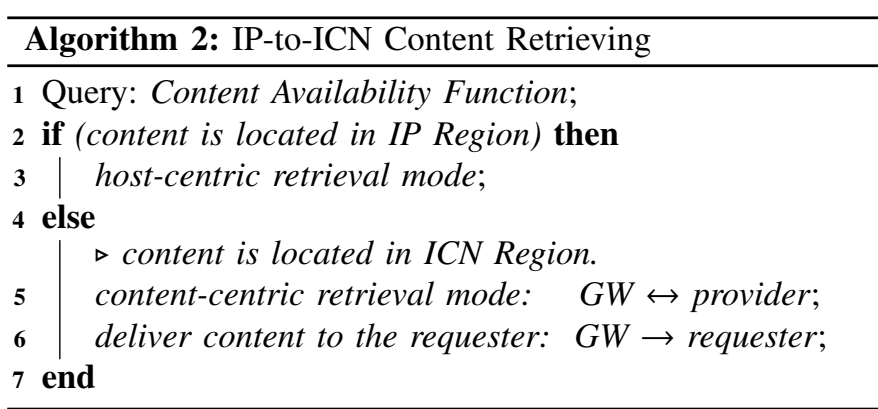

content from producer or replica node, and (b) host-centric retrieval mode: used in IP REGIONS where the provider IP address is used to fetch the content.

Taking benefits from the aforementioned control functions and the two base modes, we define two schemes to provide coexistence as shown in Figure $3 \mathrm{~b}$ and detailed as follows.

ICN-to-IP Content Retrieving: Algorithm 1 illustrates the content delivery for a request issued by an ICN node (blue box in Figure $3 b$ ). After querying the content availability function, we differentiate two cases: (i) if the content is located in an ICN REGION, then content-centric retrieval mode is used (based on content name), and (ii) if the content is located in an IP REGION, then the ICN/IP GW will retrieve the content from host who provide it using host-centric retrieval mode (based on IP addresses), and then forwarded to the ICN requester node via content-centric paradigm.

IP-to-ICN Content Retrieving: Algorithm 2 illustrates the content delivery for a request issued by an IP node (brown box in Figure 3b). After querying the content availability function, we differentiate two cases: (i) if the content is located in an IP REGION, then host-centric retrieval mode is used (based on IP addresses), and (ii) if the content is located in an ICN REGION, then the ICN/IP GW will retrieve the content from the content provider using content-centric retrieval mode (based on content name), and then forward it to the IP requester node via host-centric paradigm. 


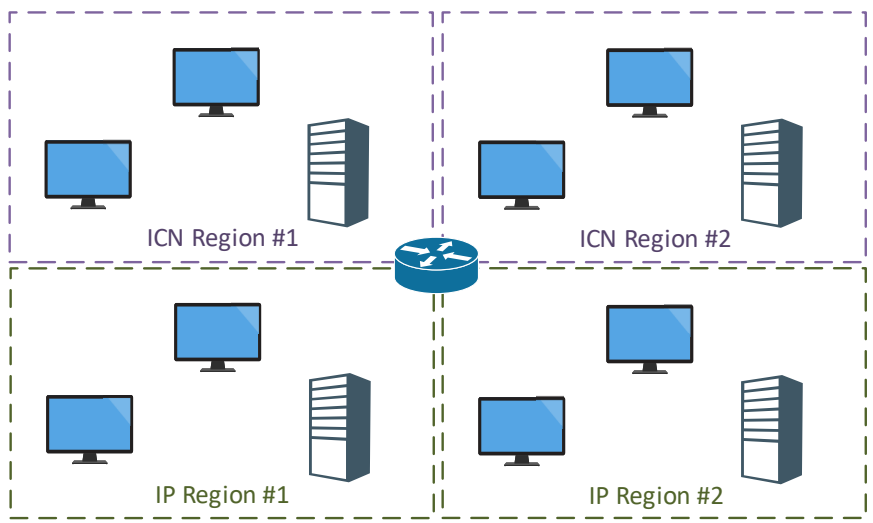

Fig. 4: Experimental Topology.

\section{EXPERIMENT EVALUATION}

We have simulated the proposed architecture on top of ndnSIM [25], which is an NS3-based simulation module for Named Data Networks. The objective is to evaluate the content delivery performance using different communication protocols. We have used a simple yet realistic topology, shown in Figure 4, with four regions (two IP REGIONS and two ICN REGIONS) interconnected via a gateway node.

The experiment consists of two phases with two scenarios in each: (i) communication within the same region, where an ICN consumer requests content from ICN provider, and an IP consumer asks from content hosted by an IP provider, and (ii) coexistence communication where an ICN consumer requests content located in an IP REGION, and an IP consumer asks for content located in an ICN REGION. The request is satisfied by downloading content with the size of 100MB with an interest rate of 10 interest/sec, we assume the system has registered more than 1000 contents.

Figure 5 shows the average network delay in different

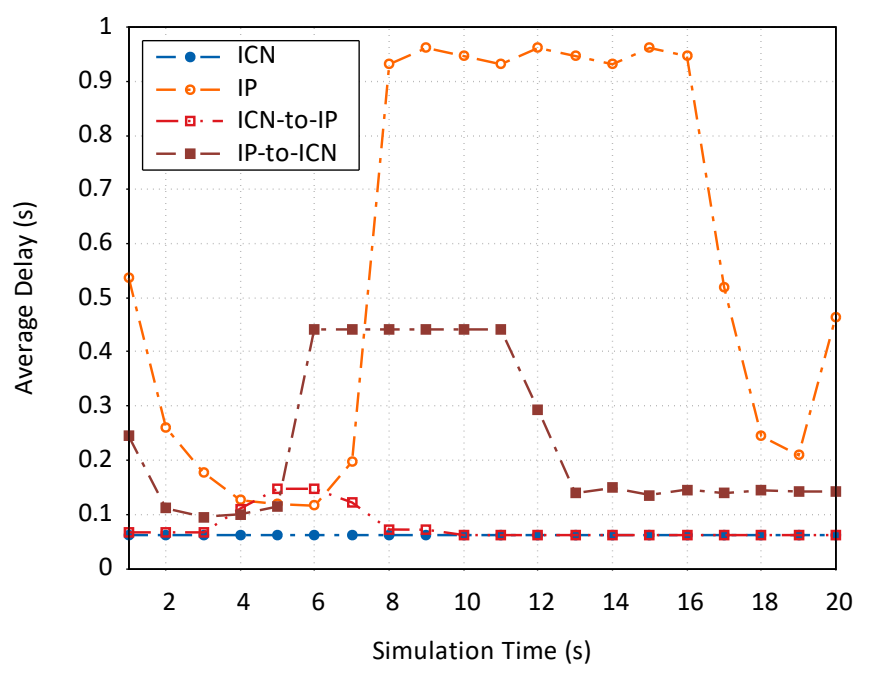

Fig. 5: Average Network Delay. scenarios. We can clearly notice that IP has the largest delay, for two reasons: the used control process to establish and manage the session, and the congestion in the network (after second 6); while ICN has the smallest delay due to the simplest packet and forwarding process without extra checking and control. On the other hand, IP-TO-ICN has an average delay with similar behavior to IP after detecting the congestion in the IP REGION. However, ICN-TO-IP has a near delay to the native ICN with a small difference in the interval [3-8] seconds. This can be justified by the fact that IP provider was located only one hop far away from the ICN/IP GW, thus the content retrieval was fast, the rest of simulation (from the ICN/IP GW to ICN consumer) was similar to the native ICN communication.

In addition, the same figure demonstrate that ICN has a better performance compared to IP networks [22]. Indeed, Figure 5 proves it by showing that ICN content retrieval is much faster than IP, with less network delay. This is due to it simple receiver-driven design, efficient forwarding scheme, and simple packet headers.

Figure 6 shows the number of packets exchanged in order to download the requested file. Both ICN-TO-IP and IP-TO-ICN require more packets compared to the base communication models due to the usage of both protocols in two regions. However, ICN requires more packets compared to IP, this can be argued to the nature of communication using Interest-Data exchange model, which means an interest is sent out to fetch a data packet while the whole content can be composed of $n$ data packets, then we have $n$ interest and $n$ data packets. However, IP can send data in multiple datagrams for one request using piggyback communication, in this scenario we have calculated also acknowledgment packets.

Moreover, the scalability and the effectiveness of the proposed solution is based on the position of the GW node. In large-scale networks, we may have multiple nodes that act as GWs. All of them are managed by the network controller.

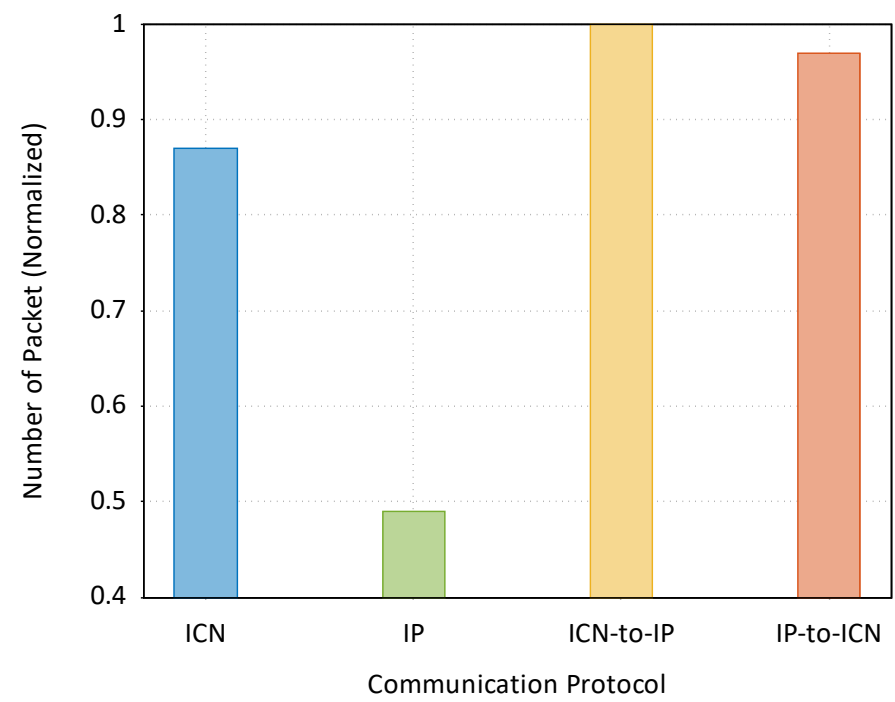

Fig. 6: Number of Packets. 
Thus, the communication between the requester, provider, and the GW can be minimized. Such a large-scale scenario will be studied in future work.

\section{Issues \& Challenges}

Several issues surround the economic factor associated with IP-to-ICN migration and coexistence such as migration cost, protocol implementation and readiness, and routing and addressing problems.

- Business Matters: if ICN deployment became necessary to provide ubiquitous content sharing and high quality of service/experience, companies that did not deploy ICN may fail to offer their services to the new market. Thus, a deep study to decide if a company/service should migrate to $\mathrm{ICN}$ is required taking into account the type of offered services and the nature of clients/consumers.

- Migration Cost: as previously discussed, deploying ICN on top of a transport protocol (e.g., TCP or UDP) may limit the performance of ICN to the performance of the under-running protocol. Hence, a clean-slate deployed is much more suitable. However, a careful study on the migration cost is extremely crucial to select which nodes will be promoted with in-network caching capabilities and ICN stack.

- Protocol Implementation: ICN migration requires not only changes in the network layer but also in the application layer to use the content name and key-word resolution instead of host addresses. The coexisting mode also requires re-implementation of the networking stack to support both protocols.

\section{CONCLUSION AND FUTURE WORK}

The deployment of ICN will overcome many issues in the current Internet. The coexistence mode is more suitable for ICN transaction with less monetary costs and risks. This paper proposed an efficient coexistence solution based on NFV technology. The approach consists of defining a set of regions and virtual control functions to assist content delivery via/cross different regions. The simulation results proved the effectiveness of the proposed approach in terms of fast content delivery and scalability. The next stage of our work will focus on extending the proposed architecture to enhance the cache placement and replacement based on the distribution of demands and content popularity, as well as improve the content forwarding based on the network's quality of service requirements.

\section{ACKNOWLEDGMENTS}

The work of F. Li was supported by the National Natural Science Foundation of China (NSFC) under Grant 61772077 and Grant 61370192. Dr. Li is corresponding author.

\section{REFERENCES}

[1] R. Mijumbi, J. Serrat, J.-L. Gorricho et al., "Network function virtualization: State-of-the-art and research challenges," IEEE Communications Surveys \& Tutorials, vol. 18, no. 1, pp. 236-262, 2016.
[2] G. Xylomenos, C. N. Ververidis, V. A. Siris et al., "A survey of information-centric networking research," IEEE Communications Surveys \& Tutorials, vol. 16, no. 2, pp. 1024-1049, 2014.

[3] B. Nour, K. Sharif, F. Li et al., "M2HAV: A Standardized ICN Naming Scheme for Wireless Devices in Internet of Things," in International Conference on Wireless Algorithms, Systems, and Applications. Springer, Jun 2017, pp. 289-301.

[4] B. Nour, K. Sharif, F. Li et al., "Security and Privacy Challenges in Information Centric Wireless IoT Networks," IEEE Security \& Privacy, 2019.

[5] L. Zhang, D. Estrin, J. Burke et al., "Named Data Networking (NDN) Project," Technical Report NDN-0001, Xerox Palo Alto Research CenterPARC, 2010.

[6] B. Nour, K. Sharif, F. Li et al., "A Survey of Internet of Things Communication using ICN: A Use Case Perspective," Computer Communications, vol. 142-143, pp. 95-123, 2019.

[7] B. Nour, K. Sharif, F. Li et al., "A Distributed ICN-based IoT Network Architecture: An Ambient Assisted Living Application Case Study," in IEEE Global Communications Conference. IEEE, Dec 2017, pp. 1-6.

[8] B. Nour, K. Sharif, F. Li et al., "NNCP: A Named Data Network Control Protocol for IoT Applications," in IEEE Conference on Standards for Communications and Networking. IEEE, Oct 2018, pp. 1-6.

[9] H. Khelifi, S. Luo, B. Nour et al., "Named Data Networking in Vehicular Ad hoc Networks: State-of-the-Art and Challenges," IEEE Communications Surveys \& Tutorials, 2019.

[10] H. Khelifi, S. Luo, B. Nour et al., "An Optimized Proactive Caching Scheme based on Mobility Prediction for Vehicular Networks," in IEEE Global Communications Conference. IEEE, Dec 2018, pp. 1-6.

[11] H. Khelifi, S. Luo, B. Nour et al., "A Name-to-Hash Encoding Scheme for Vehicular Named Data Networks," in International Wireless Communications \& Mobile Computing Conference. IEEE, Jun 2019.

[12] R. Ravindran, A. Chakraborti, S. O. Amin et al., "5G-ICN: Delivering ICN services over 5G using network slicing," IEEE Communications Magazine, vol. 55, no. 5, pp. 101-107, 2017.

[13] B. Nour, K. Sharif, F. Li et al., "NCP: A Near ICN Cache Placement Scheme for IoT-based Traffic Class," in IEEE Global Communications Conference. IEEE, Dec 2018, pp. 1-6.

[14] M. Mangili, F. Martignon, and A. Capone, "Optimal design of information centric networks," Computer Networks, vol. 91, pp. 638-653, 2015.

[15] G. Xylomenos, Y. Thomas, X. Vasilakos et al., "IP Over ICN Goes Live," in European Conference on Networks and Communications, June 2018.

[16] G. Xylomenos, A. Phinikarides, I. Doumanis et al., "IPTV Over ICN," arXiv preprint arXiv:1804.07509, 2018.

[17] S. Shailendra, B. Panigrahi, H. K. Rath et al., "A novel overlay architecture for information centric networking," in Twenty First National Conference on Communications. IEEE, Feb 2015, pp. 1-6.

[18] D. Trossen, M. J. Reed, J. Riihijärvi et al., "IP over ICN - The Better IP?" in European Conference on Networks and Communications. IEEE, June 2015, pp. 413-417.

[19] S. Shannigrahi, C. Fan, and G. White, "Bridging the ICN Deployment Gap with IPoC: An IP-over-ICN protocol for 5G Networks," $A C M$ SIGCOMM, 2018.

[20] M. Vahlenkamp, F. Schneider, D. Kutscher et al., "Enabling ICN in IP networks using SDN," in IEEE International Conference on Network Protocols, Oct 2013, pp. 1-2.

[21] P. Zuraniewski, N. van Adrichem, D. Ravesteijn et al., "Facilitating ICN Deployment with an Extended OpenFlow Protocol," in Proceedings of the 4th ACM Conference on Information-Centric Networking, 2017.

[22] I. Moiseenko and D. Oran, "TCP/ICN: carrying TCP over content centric and named data networks," in Proceedings of the 3rd ACM Conference on Information-Centric Networking. ACM, 2016, pp. 112-121.

[23] T. Refaei, J. Ma, S. Ha et al., "Integrating IP and NDN through an extensible IP-NDN gateway," in Proceedings of the 4th ACM Conference on Information-Centric Networking. ACM, 2017, pp. 224-225.

[24] S. Luo, S. Zhong, and K. Lei, "IP/NDN: A multi-level translation and migration mechanism," in NOMS - IEEE/IFIP Network Operations and Management Symposium. IEEE, April 2018, pp. 1-5.

[25] S. Mastorakis, A. Afanasyev, I. Moiseenko et al., "ndnSIM 2.0: A new version of the NDN simulator for NS-3," NDN, Technical Report NDN$0028,2015$. 